\title{
A Study of Bacteriological Profile of Burn Wound Infections in Burn Patients Admitted in a Tertiary Care Hospital in Visakhapatnam, India
}

\author{
T. Parvathi, T. Aditya* and P. Appa Rao \\ Department of Microbiology, Andhra Medical College, Visakhapatnam, \\ Andhra Pradesh, India \\ *Corresponding author
}

\section{Keywords \\ Burn wound infection, \\ Bacteriological profile, Antibiotic susceptibility pattern, Hospital environment}

\section{Article Info}

Accepted:

17 October 2019

Available Online:

10 November 2019

\section{A B S T R A C T}

A descriptive study was carried out among 100 patients admitted in burns ward in a tertiary care hospital, Visakhapatnam over a period of one year from January 2018 to December 2018. The aim of the study is to determine the bacterial etiological agents prevalent in burn wound infection and their antimicrobial sensitivity pattern. Material methods - A total of 200 swabs were collected from patients with burn wound infection and cultured using standard aerobic microbiological techniques. Antimicrobial susceptibility testing was done for different agents using Kirby Bauer disc diffusion method. Results- out of the 200 swabs cultured from burn wound infections, 190 swabs were culture positive and 10 swabs were found to be sterile. The isolation rate was $95 \%$. A predesigned pro-forma was made to show variables which include patient occupation, age, sex, percentage of burn, depth of the burn, site from where culture was taken, day of the swab taken, and culture report. Culture from burn wound infection revealed that Pseudomonas aeruginosa was the most common organism (36\%) followed by staphylococcus aureus (28.5\%) and other organisms include E.coli (8\%), Klebsiella sp. $(21 \%)$, Acinetobacter sp. (1.5\%). There was a high rate of multi drug resistant organisms. Out of the 100 swabs taken on the $3^{\text {rd }}$ day of admission i.e. $1^{\text {st }}$ isolate, the most common organism isolated was Staphylococcus aureus (27\%), followed by Pseudomonas aeruginosa (26\%), Klebsiella (22\%), E.coli (12\%), Acinetobacter species (3\%). 10 swabs were found to be sterile. Staphylococcus aureus was sensitive to Linezolid (100\%), Amoxyclave (81\%), Clindamycin (88\%), Erythromycin (66\%), Cotrimaxazole (66\%), Cefoxitin (77\%), Amikacin (12\%), and Tetracycline (51\%). Gram negative isolates were sensitive to Pipercillin+Tazobactam (100\%), Meropenem (100\%), Cefepime (74\%), Ceftazidime+Clavulanicacid (100\%), Gentamicin (15\%), Amikacin (38\%), and Cotrimaxazole $(19 \%)$. Out of the 100 culture taken on the $5^{\text {th }}$ day of admission i.e. 2 nd isolate the most common organism isolated was Pseudomonas aeruginosa (46\%), Staphylococcus aureus (30\%), Klebsiella sp. (20\%), E. coli (4\%). Gram negative isolates were sensitive to Piperacillin+Tazobactam (100\%), Meropenem (100\%), Ceftazidime+Clavulanicacid (100\%), Cefepime (88\%), Gentamicin (31\%), and Amikacin (42\%). Staphylococcus aureus was sensitive to Linezolid (100\%), Clindamycin (86\%), Cefoxitin (86\%), Amoxyclave (66\%), Erythromycin (53\%), and Tetracycline (56\%). Conclusion-The study showed majority of the patients were from the rural area of which female $(65 \%)$ patients were more in number. Collection of appropriate samples for culture before starting of antibiotics helps better management of patients along with isolation precautions. Close follow up of patients and repeat isolations are necessary for appropriate change in antibiotics. Careful selection of antibiotics to control the wound infection is required for proper management of burn wound infection in order to help reduce morbidity and mortality. 


\section{Introduction}

Infection remains the leading cause of death among patients who are hospitalized for burns. Thermal injury destroy the skin barrier and make the burn wound the most frequent origin of sepsis in these patients [1]. Initially the burn area is considered free of microbial contamination. The risk of burn wound infection is directly proportional to the extent of burn and related to impaired resistance. Gram positive bacteria heavily colonize the wound within 48 hours of injury. Topical antimicrobials decrease the bacterial over growth. The potentially invasive bacterial pathogens derived from patient's gastrointestinal tract and upper respiratory tract and hospital environment [1.11].

Globally burns are considered devastating forms of trauma to serious thermal injury. Burns could be caused by scalds, thermal, electrical, gas and chemical agents [2.3]. Patients with serious burn injury require immediate specialized care in order to minimize bacterial infection which is a major cause of morbidity and mortality [4.5]. Although in most cases the source of contamination is patient's normal flora or exogenous contamination from contaminated wound dressing device or from hospital environment. Various groups of microorganisms have been reported to be associated with wound infection [6.7]. A study carried out by Patil et al., [7] in India revealed that Pseudomonas aeruginosa, Staphylococcus aureus, Acinetobacter baumanii, Klebsiella pneumonia, Proteus mirabilis, Citrobacter sp, Enterobacter sp., Escherichia coli were commonly associated with burn wound infection. Antibiotic susceptibility pattern for microorganisms isolated from hospitalized patients are continuously evolving and this can pose a major challenge for clinicians treating burn wound victims [8.9.12]. The study was conducted to determine microbial profile of burn wound infection in respect to age, sex, occupation among burn wound injured patients in a tertiary care hospital, Visakhapatnam.

\section{Materials and Methods}

The swabs were taken from the infected areas that appeared deep with discharge. The swabs were taken on 3rd and 5th day of admission. After cleaning properly with normal saline, the swabs moistened with sterile saline rubbed over the wound surface. The swabs were immediately transported to microbiology laboratory. In the lab the swabs were cultured on to blood agar, MacConkey agar plates and incubated at $37^{\circ} \mathrm{C}$. After overnight incubation the colony morphology, color, shape, pigmentation examined. A single colony from blood agar and MacConkey agar was taken and Gram stained and then identified by biochemical reaction like IMViC tests and sugar fermentation, TSI, urease, oxidase and catalase tests were done to identify the bacterial species. Antimicrobial susceptibility testing was done by suspending individual colonies in saline by matching to a turbidity equivalent of 0.5 McFarland standard. The suspensions were then streaked onto Muller Hilton agar plate using sterile swab sticks. The antibiotic paper disks were gently but firmly placed on the inoculated plates using sterile forceps. The plates were incubated at $37^{\circ} \mathrm{C}$ overnight and zones of inhibition was measured and interpreted according to CLSI and EUCAST guidelines [10.11]. The antibiotic discs used are Piperacillin+Tazobactam, Gentamicin, Amikacin, Linezolid, Meropenem, Ceftazidime+Clavulanicacid, Cefepime, Amoxyclave, Erythromicin, Cotrimaxazole, Tetracycline and Clindamycin.

The data obtained from the study were analyzed using descriptive statistics generated with the help of Microsoft Excel. Quantitative 
data is analyzed using SPSS version 22. In all cases p-value less than 0.005 were considered statistically significant. Participation by patients was voluntary in accordance with Ethics committee guidelines.

\section{Results and Discussion}

A total of 200 swabs were taken from patients admitted in burn unit over a period of one year and were processed to identify bacteriological profile and their antimicrobial susceptibility pattern. Out of the 200 swabs, 190 swabs revealed culture positive, while 10 swabs showed sterile. The overall isolation rate was $95 \%$.The sex ratio showed female $(65 \%)$ preponderance over males $(35 \%)$. Most of the studied group belonged to housewives $(48 \%)$ followed by daily wage laborers (34\%). Majority of the patients $(32 \%)$ had more than $50 \%$ of burns. Most of the patients had full thickness burn (58\%). Most common age group involved was between 31-40 years $(38 \%)$. Most of the culture swabs were taken from abdomen (65\%) followed by back, extremities, thorax, and head and neck.

Among the 200 swabs collected in the present study, bacterial isolates identified were 72 Pseudomonas aeruginosa (36\%), 57 Staphylococcus aureus (28.5\%), followed by 42 Klebsiella (21\%),16 E.coli (8\%), 3 Acinetobacter $(1.5 \%)$ and 10 swabs were sterile( $5 \%)$.

All gram negative bacteria were sensitive to Piperacillin+Tazobactam, Meropenum, Ceftazidime+Clavulanicacid, Cefepime, Gentamicin and Staphylococcus aureus was sensitive to Linezolid, Clindamycin, Amoxyclave, Cefoxitin, Erythromycin, and Cotrimaxazole.10 Staphylococcus aureus were found to be MRSA strains.

During the study period of one year a total of 100 patients were taken for study, from them
200 swabs were collected, the isolation rate is 95\% which is comparable with Vandana et al., $(91.27 \%)[13]$. The sex ratio showed female $(65 \%)$ preponderance over males $(35 \%)$ this can be attributed to females being exposed to household fire while cooking and also suicidal and dowry deaths. This finding in burn patients correlated with Nazreen A. Siddique et al., Gupta et al., Singh et al., [14,15,16]. Most of the patients belong to low socio economic groups and most of them were from rural areas.

The incidence of burns was highest among the age group of 31 to 40 years $(31 \%)$ followed by age group 21 to 30 years (24\%). In the present study most of the patients who developed infection had burns of more than 50\% TBSA therefore the more was the TBSA, the more was the risk of colonization and proliferation of various bacteria in burn wound. Most patients had full thickness burns (55\%), most cultures were taken were from the abdomen $(65 \%)$.

In the present study we found that the most frequent isolates were Pseudomonas aeruginosa (36\%) followed by Staphylococcus aureus (28.5\%), Klebsiella (21\%), E.coli $(8 \%)$ and Acinetobacter species (1.5\%). Similar studies conducted by Sewunet et al., [17], Biswal et al., and Rajput et al., [18,19.] also revealed that Pseudomonas was the most predominant organism. Pseudomonas was sensitive to antibiotics like Piperacillin+ Tazobactam, Meropenem, Ceftazidime+ Clavulanicacid, and Cefepime. Staphylococcus aureus was sensitive to Linezolid, Cefoxitin, Amoxyclave, Clindamycin, Erythromycin and Cotrimaxazole. We also found that there was a change in bacterial flora with time, in the first isolates Staphylococcus aureus was the most common micro organism isolated while in the second isolates, Pseudomonas aeruginosa was the predominant one (Fig. 1 and 2; Table 1-4). 
Table.1 Guidelines for interpreting antimicrobial susceptibility results

\begin{tabular}{|c|c|cc|}
\hline Antibiotic & Disk Content $\mu \mathrm{g}$ & \multicolumn{2}{|c|}{ Zone diameter in $\mathrm{mm}$} \\
$\mathrm{S}>=$ & $\mathrm{R}<=$ \\
\hline Erythromycin & & $>23$ & $<14$ \\
\hline Amoxycillin+Clavulanicacid & 15 & $>18$ & $<13$ \\
\hline Clindamycin & $20 / 10$ & $>21$ & $<14$ \\
\hline Gentamicin & 2 & $>15$ & $<14$ \\
\hline Amikacin & 10 & $>17$ & $<14$ \\
\hline Cefoxitin & 30 & $>18$ & $<18$ \\
\hline Cefepime & 30 & $>25$ & $<20$ \\
\hline Ceftazidime+Clavulanicacid & 30 & $>27$ & $<10$ \\
\hline Cotrimaxazole & $30 / 10$ & $>16$ & $<14$ \\
\hline Linezolid & 25 & $>21$ & $<15$ \\
\hline Tetracycline & 30 & $>19$ & $<17$ \\
\hline Meropenem & 30 & $>23$ & $>21$ \\
\hline Piperacillin+Tazobactam & 10 & & \\
\hline & $100 / 10$ & & \\
\hline
\end{tabular}

Table.2 First Isolate $3^{\text {rd }}$ Day

Second isolate $5^{\text {th }}$ Day

\begin{tabular}{|c|c|c|c|}
\hline Organism & Number(n) & Organism & $\begin{array}{c}\text { Number } \\
\text { (n) }\end{array}$ \\
\hline Staphylococcus aureus & 27 & Pseudomonas aeruginosa & 46 \\
\hline Pseudomonas aeruginosa & 26 & Staphylococcus aureus & 30 \\
\hline Klebsiella sp. & 22 & Klebsiella $s p$ & 20 \\
\hline Escherichia coli & 12 & Escherichia coli & 4 \\
\hline Sterile & 10 & & \\
\hline
\end{tabular}

Table.3 Antibiotic sensitivity pattern of $1^{\text {st }}$ isolate

\begin{tabular}{|c|c|c|c|c|c|c|c|c|}
\hline Gram Positive & AK & AMC & $\mathbf{E}$ & CL & COT & CX & LZ & TE \\
\hline $\begin{array}{l}\text { Staphylococcus } \\
\text { aureus } n=27\end{array}$ & $11 / 16$ & $22 / 5$ & $18 / 9$ & $23 / 3$ & $18 / 9$ & $21 / 6$ & 27 & $14 / 13$ \\
\hline Gram Negative & G & PIT & AK & CF & MEP & CTC & COT & \\
\hline $\begin{array}{c}\text { Pseudomonas } \\
\text { aeruginosa } \mathrm{n}=26\end{array}$ & $3 / 23$ & 26 & $12 / 14$ & $20 / 6$ & 26 & 26 & $5 / 21$ & \\
\hline Klebsiella $\mathrm{n}=\mathbf{2 2}$ & $6 / 16$ & 22 & $8 / 14$ & $18 / 4$ & 22 & 22 & $3 / 19$ & \\
\hline Escherichia coli $\mathrm{n}=12$ & $1 / 11$ & 12 & $3 / 9$ & $8 / 4$ & 12 & 12 & $4 / 8$ & \\
\hline Acinetobacter $\mathrm{n}=3$ & 0 & 3 & $1 / 2$ & $1 / 2$ & 3 & $2 / 1$ & 0 & \\
\hline
\end{tabular}


Table.4 Antimicrobial susceptibility pattern of $2^{\text {nd }}$ isolate

\begin{tabular}{|c|c|c|c|c|c|c|c|c|}
\hline Gram Positive & AK & AMC & E & CL & COT & CX & LZ & TE \\
\hline $\begin{array}{c}\text { Staphylococcus } \\
\text { aureus n=30 }\end{array}$ & $11 / 19$ & $20 / 10$ & $16 / 14$ & $26 / 4$ & $14 / 16$ & $26 / 4$ & 30 & $17 / 13$ \\
\hline $\begin{array}{c}\text { Gram Negative } \\
\text { Pseudomonas } \\
\text { aeruginosa } \\
\text { n=46 }\end{array}$ & $18 / 28$ & 46 & $22 / 24$ & $38 / 8$ & 46 & 46 & $12 / 34$ & \\
\hline $\begin{array}{c}\text { Klebsiella } \text { n=20 } \\
\text { Escherichia coli }\end{array}$ & $3 / 17$ & 20 & $8 / 12$ & 20 & 20 & 20 & $6 / 14$ & \\
$\mathbf{n = 4}$ & $1 / 3$ & 4 & 0 & 4 & 4 & 4 & 0 & \\
\hline
\end{tabular}

Fig.1

\begin{tabular}{|c|c|c|}
\hline \multicolumn{2}{|c|}{ First Isolate } & Second Isolate \\
\hline - Staphylococcus aureus & | Pyeudomonas aeruginosa & - Pseudomonas aeruginosa | Staphylococcus aureus \\
\hline = Klebsiella & "Escherichia coli & "Escherichia Coli \\
\hline - Acinetobacter & " Sterile & $4 \%$ \\
\hline $3 \times 1$ & $2 \pi \%$ & \\
\hline
\end{tabular}

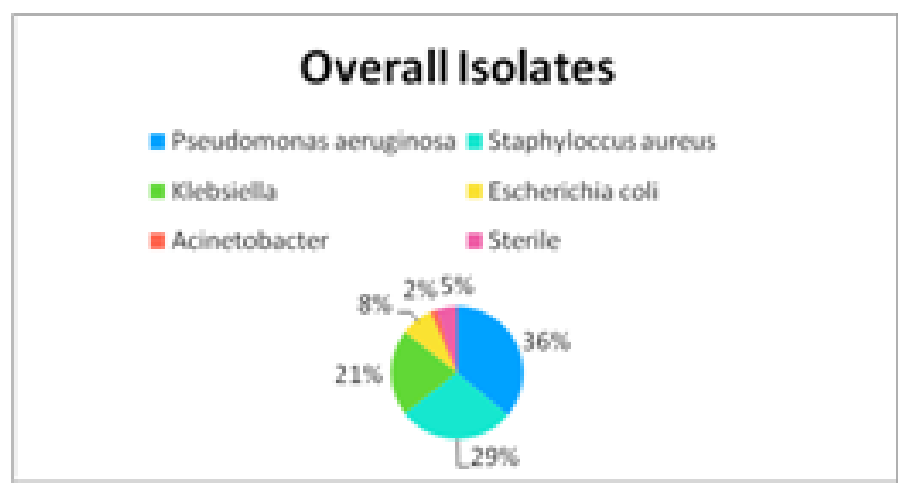


Fig.2 Change in the role of isolates

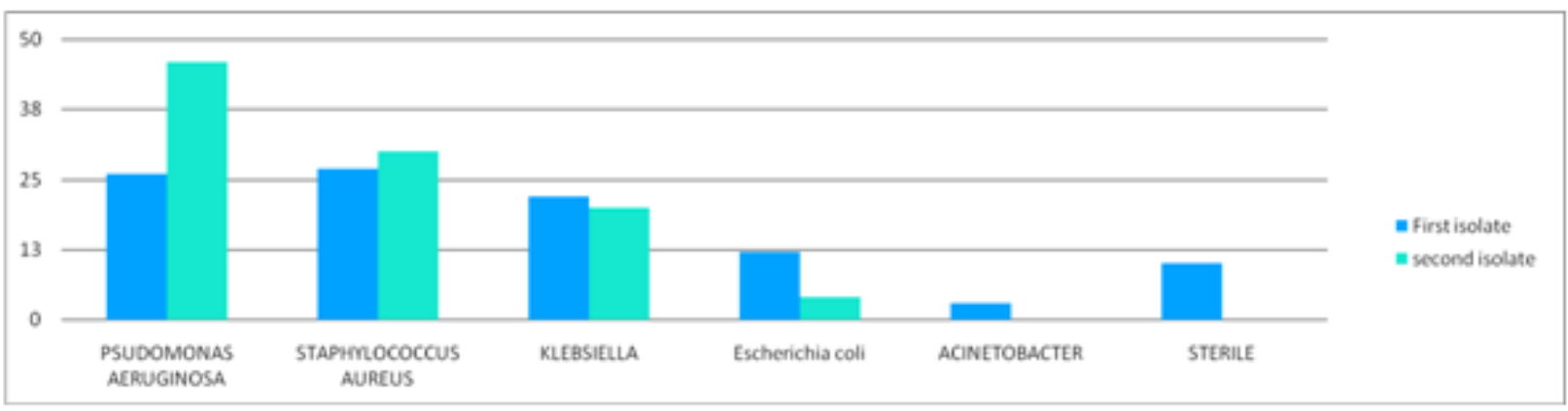

Out of the 200 swabs taken, 10 cultures were found to be sterile all of which belong to the first isolate which was the similar to the study conducted by Vandana et al., [13]. There were also changes in the pattern of bacterial sensitivities to the antibiotic studied; the second isolates were more sensitive to Piperacillin+Tazobactam and Meropenem than the first isolates.

Hence frequent evaluation of microbes and their antibiotic susceptibility pattern remain the urgent need of a burn unit to safe guard the patients against the life threatening infections. The use of antibiotics should be made rotational to get rid of the resistance produced in the microbes against common antibiotics.

My study will be helpful in providing useful guidelines for choosing effective empirical therapy which will have great impact on morbidity and mortality of burn patients due to bacterial infection or septicemia and also hospital environmental microbial flora, their sensitivity pattern should be studied in every burn unit in order to prevent the emergence of multi-drug resistant strains, because of indiscriminate use of antibiotics

\section{References}

Branski LK1, Al-Mousawi A, Rivero H, Jeschke MG, Sanford AP, Herndon DN. Emerging infections in burns.
2009 Oct; 10(5):389-97. doi: 10.1089/sur.2009.024.

Clinical and Laboratory Standards Institute.

Performance Standards for

Antimicrobial Disk Susceptibility Tests; Approved Standard-Twelfth Edition. Volume 35 Number 1 https://clsi.org/media/1631/m02a12_sa mple.pdf

Dalbir Singha Amarjit Singhb Aditya K. Sharmaa Lavina Sodhia. Burn mortality in Chandigarh zone: 25 years autopsy experience from a tertiary care hospital of India. Burns, Volume 24, Issue 2, March 1998, Pages 150-156.

Gupta RK1, Srivastava AK. Study of fatal burns cases in Kanpur (India). Forensic Sci Int. 1988 Apr; 37(2): 81-9. https://www.ncbi.nlm.nih.gov/pubmed/ 3371805

Hansbrough JF1, Wikström T, Braide M, Tenenhaus M, Rennekampff $\mathrm{OH}$, Kiessig V, Bjursten LM.Neutrophil activation and tissue neutrophil sequestration in a rat model of thermal injury. J Surg Res. 1996 Feb 15; 61(1):17-22.

Indu Biswal, Balvinder Singh Arora, Dimple Kasana and Neetushree. Incidence of Multidrug Resistant Pseudomonas aeruginosa Isolated from Burn Patients and Environment of Teaching Institution. J Clin Diagn Res. 2014 May; 8(5): DC26-DC29 
Jefferson Lessa Soares de Macedo, João Barberino Santos. Bacterial and fungal colonization of burn wounds. Mem Inst Oswaldo Cruz, Rio de Janeiro, Vol. 100(5): 535-539, August 2005

Monafo WW, Freedman B. Topical therapy for burns. Surg Clinic North America 1987 Feb; 67(1): 133-45

Neriman Akansel, Nurşen Görgeç, Sevinç Yılmaz, Ramazan Kahveci. Burn Wound Infections in Medical Hospital Burn Unit in Bursa, Turkey. International Journal of Caring Sciences September-December 2014 Volume 7 Issue 3 776-780

Patil P, Joshi S, Bharadwaj R. Aerobic bacterial infections in a burns unit of Sassoon General Hospital, Pune. International J. of Healthcare and Biomedical Research, Volume: 03, Issue: 03, April 2015, Pages 106-112

Rajput A, Saxena R, Singh KP, Kumar V, Singh S, Gupta A, Singh RK. Prevalence and antibiotic resistance pattern of metallo-beta-lactamaseproducing Pseudomonas aeruginosa from burn patients--experience of an Indian tertiary care hospital. J Burn Care Res. 2010 Mar-Apr; 31(2) :264-8.

Sayoni Datta, Tapashi Ghosh, Devleena Sarkar, Nikhil Kumar Tudu, Tapan Kumar Chatterjee, Angshuman Jana. Bacteriological Profile of Burn Wounds and Their Antibiotic Susceptibility Pattern in a Tertiary Care Hospital. International Journal of Scientific Study. 2016. 4(5): 141-145.

Seyyed Jalil Mirmohammadi, Amir Houshang Mehrparvar, Kazem Kazemeini,1 and
Mehrdad Mostaghaci.Epidemiologic Characteristics of Occupational Burns in Yazd, Iran. Int J Prev Med. 2013 Jun; 4(6): 723-7.

Shittu A.O., Kolawole D.O. and OYEDEPO E.A.R..A Study of wound infections in two health institutions in ILE-IFE, Nigeria. Afr. J. Biomed. Res. Vol. 5; $97-102$.

Siddique NA. Burn injury is preventable. An analysis of 716 cases in a burn unit. JCPSP 1998; 8: 148-152.

Tsegaye Sewunet, Yohanes Demissie, Adane Mihret, Tamrat B Abebe. Bacterial Profile and Antimicrobial Susceptibility Pattern of Isolates Among Burn Patients at Yekatit 12 Hospital Burn Center, Addis Ababa, Ethiopia Ethiop J Health Sci. 2013 Nov; 23(3): 209-16. https://www.ncbi.nlm.nih.gov/pubmed/ 24307820

Vandana Berry and Vidya Sagar. 2006. Bacteriology of burn wounds as assessed in Christian Medical College and Hospital, Ludhiana. Journal of Medical Science 2006:81-84.

World Health Organisation (10/01/2017): Burn prevention success stories and lessons learned https://www.who.int/ violence_injury_prevention/publicatio ns/other_injury/burn_success_stories/e $\mathrm{n} /$

Yusuf Kenan Coban. Infection control in severely burned patients World J Crit Care Med. 2012 Aug 4; 1(4):94-101. doi: $\quad 10.5492 /$ wjccm.v1.i4.94. eCollection 2012 Aug 4.

\section{How to cite this article:}

Parvathi, T., T. Aditya and Appa Rao, P. 2019. A Study of Bacteriological Profile of Burn Wound Infections in Burn Patients Admitted in a Tertiary Care Hospital in Visakhapatnam, India. Int.J.Curr.Microbiol.App.Sci. 8(11): 1932-1938. doi: https://doi.org/10.20546/ijcmas.2019.811.227 